has been the object of some controversy. It is generally thought that the southward turning of the field leads directly to a series of growth processes which trigger the substorm. On the other hand measurements in Alaska indicate that substorms occur frequently even when the field component is directed northwards. This conflict has been resolved by Akasofu (Nature, 286, 191; 1975) who suggests that the occurrence of a substorm depends neither on the field direction nor on its rate of change, except that if a steady northward field $(>6 \mathrm{~h})$ is established then no substorms are observed. During these events energetic particles travelling along the magnetic field lines penetrate into the lower ionosphere where they produce ionisation enhancements in both $\mathrm{D}$ and $\mathrm{E}$ regions which greatly affect the propagation of radio waves through these regions. It has been suggested that even lower levels of the atmosphere are influenced by geomagnetic storms. Olson (Nature, 257,$113 ; 1975$ ) has found that at tropospheric heights, the 500 mbar vorticity index, which is a rough measure of cyclonic activity in the Northern hemisphere, decreases during the main phase of a storm.

Storm-induced disturbances are not confined to polar latitudes and the $F$ region of the ionosphere is affected even at the magnetic equator. Both increases and decreases in this layer's electron density are observed depending on the particular storm event. The detailed mechanisms of $F$ region storms have been discussed by Rishbeth (J. atmos. terr. Phys., 37, 1055; 1975) and changes in thermospheric circulation are shown to be a major factor in producing the observed ionospheric disturbances. In principle, both positive and negative storms can be caused by circulation changes though it is likely that other factors such as electromagnetic drift, ionosphere-magnetosphere plasma flux and the effects of propagating gravity waves have important roles especially at low latitudes. Storm-induced exchanges of ionisation between the ionosphere and protosphere have been observed by Soicher in a recent satellite experiment (page 33, this issue).

In view of the complexity of the storm processes there is an urgent need for further measurements of particle fluxes, magnetic field variations and ionisation changes. An international cooperative effort to obtain these observations will be made during the forthcoming International Magnetosphere Study (IMS), period 1976 to 1978. A wide range of ground based and space vehicle techniques will be deployed in a coordinated programme to study the magnetosphere and its influences on other regions of the Earth's environment.

\section{Weak gamma-ray bursts}

\section{from A. C. Fabian}

SOURCE counts are widely used by astronomers working in all parts of the spectrum, and appear under various names and guises. Comparisons of the number of faint sources with brighter ones can yield information on the geometrical distribution of sources in space, their evolution with time, cosmological effects and the distribution of absorbing material. This information is gained without any precise knowledge of the distances to the sources. Unfortunately, however, source counts do not give unique solutions to problems. This is mostly due to the fact that there is always some spread in the intrinsic luminosities of the sources.

The latest category of events to be counted are cosmic gamma-ray bursts. These are the enigmatic flashes of gamma radiation first detected by the Vela satellites. The only facts really known about them are that they show complex structure on time scales of hundredths of seconds to tens of seconds, their spectra somewhat resemble those expected from thermal bremsstrahlung from regions of temperature $\sim 10^{\circ} \mathrm{K}$, and their origins lie outside the inner Solar System. Counts of the more intense bursts detected by the Vela and other satellites, with integrated fluxes greater than $\sim 10^{-4} \mathrm{erg}$ $\mathrm{cm}^{-2}$, suggest that they are relatively uniformly distributed in space. The rate is such that weaker events $\left(\sim 10^{-7}\right.$ erg $\mathrm{cm}^{-2}$ ) are predicted to occur several times per hour. Such a prediction is testable by balloon-borne detectors, and Bewick, Coe, Mills and Quenby of Imperial College London have recently reported the results of a series of flights (Nature, 258, 686; 1975).

Only one event was observed, of size $1.2 \times 10^{-7} \mathrm{erg} \mathrm{cm}^{-2}$, in $13.5 \mathrm{~h}$ of observing time. This is a significantly different rate to that predicted. In the authors' opinion it suggests that a uniform source distribution can be excluded. Such a result might be expected if the sources are galactic. for then there might be fewer weaker sources observed in directions perpendicular to the galactic plane. Cosmological effects-principally redshiftmight be invoked if the sources of gamma-ray bursts are extragalactic. Some caution is appropriate in interpreting the results, however, since event rates are being compared between different detectors of somewhat uncertain apertures, efficiencies and energy ranges. Consequently there are errors in the integrated fluxes that are difficult to estimate. The Imperial College detectors appear to be sensitive enough to avoid limitations due to the transient nature of the bursts. This would only become serious if a substantial fraction of the bursts had timescales of $10 \mathrm{~s}$ or more, but there is at present no indication of this being so.

There are undoubtedly many other groups building or flying balloon-borne detectors to monitor weak gamma-ray bursts. It is hoped that these will confirm the first results and will eventually define the shape of the size distribution of weak gamma-ray bursts. The rare stronger bursts will probably only be available for study by satellite detectors. We are still no closer to a theoretical interpretation of gammaray bursts, although it is notable that the number of theories proposed to explain them is now lagging behind the number of detected events.

\section{Fish that change sex}

\section{from John R. Krebs}

To a narrow-minded mammal the sex life of the Blue-headed Wrasse, a tropical reef fish, may seem odd. The Blue-headed Wrasse occurs in two colour phases: smaller fish are highly variable in colour and may be either male or female; while larger, older individuals are always blue and green with two vertical black stripes, and are invariably male. Some individuals go through the first (initial phase) colour pattern as a female and turn into a male when they reach the striking terminal colour phase. Other individuals go through both phases as a male. The transition from initial to terminal colour phase and the accompanying sex change can be achieved rapidly. If all the terminal phase males are taken away from an area of reef, initial phase males or females soon start to change colour and become terminal males.

Most of the fertilising of females is done by terminal males. They set up temporary territories at around midday at a specific spawning site on the reef. Here they wait for females to turn up, while the smaller initial phase males mill around in large numbers (often in hundreds). When females arrive at the spawning area they prefer to mate with the territorial terminal males, and a lucky male may mate up to 100 times a day. Initial phase males sometimes manage to fertilise a 